\title{
Obtaining non-spherical poly(alkylcyanoacrylate) nanoparticles by the stretching method applied with a marketed water-soluble film.
}

\author{
Claudio Palazzo, ${ }^{1,2+}$ Gilles Ponchel, ${ }^{2}$ Jean Jacques Vachon, ${ }^{2}$ Sarah Villebrun, ${ }^{2}$ Florence \\ Agnely, ${ }^{2}$ Christine Vauthier ${ }^{2 *}$ \\ ${ }^{1}$ Institut Galien Paris Sud, CNRS UMR 8612, Univ. Paris-Sud, Université Paris-Saclay, Chatenay- \\ Malabry, France \\ 2Dipartimento di Farmacia-Scienze del Farmaco, Università degli Studi di Bari "Aldo Moro", Via \\ Orabona, 4 - 70125 Bari, Italy
}

Short title: Making elongated PIBCA nanoparticles by stretching

Published in: Int J Polym Mater 2017;66:416-424. https://doi.org/10.1080/00914037.2016.1233420

*Corresponding author

Christine Vauthier, Institut Galien Paris Sud, CNRS UMR 8612, Univ. Paris-Sud, Université ParisSaclay, 5 Rue J.B. Clément, 92296 Chatenay-Malabry Cedex, France

E-mail address: christine.vauthier@u-psud.fr, Phone 33146835603 / Fax : 33146835946

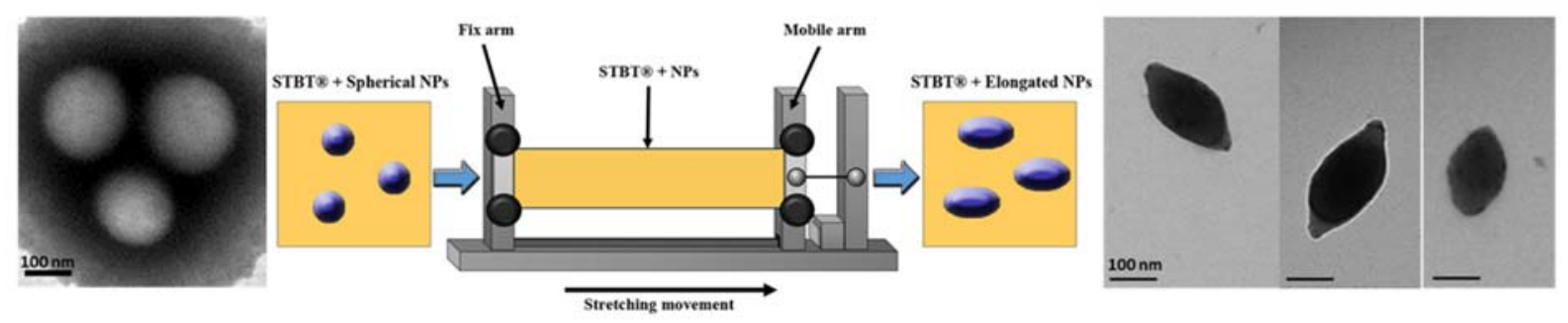

Abstract: This work was aimed to produce elongated nanoparticles of clinical interest by the stretching method. Polymer films prepared from material recovered from the dissolution of a marketed stretchable water-soluble film were loaded with $10 \%$ of poly(isobutylcyanoacrylate) nanoparticles. The films were elongated at $50^{\circ} \mathrm{C}$ to $230 \%$ of their initial lengths. The shape factor of the nanoparticles recovered after elongation was consistent with stretching conditions applied on the film. This work has demonstrated the feasibility of the preparation of non-spherical poly(isobutylcyanoacrylate) nanoparticles by the stretching method and described conditions to apply the method.

Key words: stretching, nanoparticles, shape factor, polymer film, poly(isobutylcyanoacrylate) 


\section{Introduction}

Encapsulation of drugs in polymeric carriers is a widely investigated strategy aiming to manage stability concerns and/or inadequate biodistribution of unprotected drugs in the body [1]. In the last decades, a step by step accumulation of knowledge have shown that modulation of their physicochemical characteristics, including size and chemical surface composition, could strongly impact the in vivo fate of nanoparticles in the body [2-5]. Conversely, only very few experimental reports have investigated so far the influence of the shape of nanoparticles on their biodistribution [6] and phagocytosis by cells [7-11]. Commonly used nanoparticle preparation methods could only generate sphere-shaped nanoparticles [12-14] while the obtaining of non-spherical shapes polymeric nanoparticles was described more recently $[4,7,8,15-17]$. First investigations of their in vivo fate have revealed that elongated nanoparticles behaved markedly differently from their corresponding spherical counterparts $[\mathbf{6 - 8 , 1 0 , 1 8 - 2 3 ]}$. Thus shape has become a new parameter to consider while designing nanoparticles for drug delivery. However, only few methods of fabrication of nanoparticles with shapes that differ significantly from a sphere have been proposed so far, each with pros and cons. The PRINT method is one of them, which has been initially proposed for the preparation of non-spherical microspheres. Basically, it consists in molding a polymer in a hard material template, followed by a recovery process. In theory, it allows the generation of particles with many different shapes that are defined by the shape of the template [16]. Because of the process it-self, the shape and size characteristics of the population of particle produced are expected to be highly homogeneous. This method was used to produce nanocubes and nanorods. Alternatively, nanoparticles with an elongated shape could be generated by the method of nanoprecipitation which is widely used to produce spherical nanoparticles from polymers $[6,24]$. However, it requires the use of specifically conformed polymers, including a few polypeptides, able to self-associate together to form crystalline domains that are believed to give an elongated shape to nanoparticles [24]. Alternatively, elongated nanoparticles were also produced by stretching spherical nanoparticles, which can be previously prepared by a suitable method [4,6-8]. Although obtaining non spherical nanoparticles by the nanoprecipitation method is reserved to a few types of polymers having the right characteristics as explained above, the application of the stretching method may be suitable to obtain non-spherical nanoparticles made of wide range of polymers. It can be assumed that this method could be used to produce homogeneous series of elongated nanoparticles differing only from their shape as produced from the same parent spherical nanoparticles. The obtaining of consistent series of nanoparticles with different shapes are required to evaluate the influence of the shape factor of the nanoparticles on their in vivo fate to help in the understanding of the effect of 
the shape of nanoparticles on their interactions with cells and their capacity to go across different biological barriers.

The aim of the present work was to set up experimental conditions to prepare elongated nanoparticles of poly(alkylcyanoacrylate) nanoparticles from parent spherical nanoparticles applying the stretching method. The parent nanoparticles used in the present study were selected for several reasons. They can be prepared with different characteristics in a well-controlled manner by highly reproducible methods of emulsion polymerization [25-30]. Their interest to improve in vivo delivery of drug is well documented considering a wide range of drug and therapeutic applications [31-38]. They are one of the very few nanomedicines made of polymer nanoparticles that are considered in an ongoing clinical trial phase II/III [34-36]. Additionally, poly(alkylcyanoacrylate) nanoparticles were developed as model nanoparticles to investigate the influence of the size and surface properties on interactions with biological systems and in turn on their in vivo fate [27,39-42]. Having the corresponding elongated species of this series would provide with the required model nanoparticles to also investigate the influence of the nanoparticle shape. The chitosan-coated poly(isobutylcyanoacrylate) (PIBCA) nanoparticles were selected to carry on the present work. These nanoparticles were designed as mucoadhesive drug delivery systems to promote oral administration of drugs [28]. They were also tested as an antitumor treatment based on the delivery of siRNA by intravenous injection $[37,38,43]$.

The stretching method was applied to produce elongated micro- and nanoparticles from their

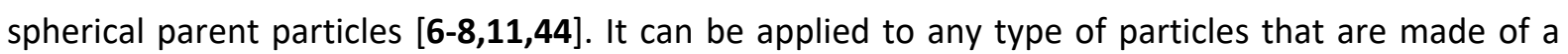
material that can be soften at the operating temperature of the stretching machine. Elongated microparticles made of both non-biodegradable and biodegradable polymers have been produced. In contrast, to the best of our knowledge, the method was only applied with nanoparticles made of non-biodegradable polymers (i.e. polystyrene) [11]. The method was not applied yet to the production of elongated biodegradable particles in the nano-size range. Interestingly, the stretching method can be scaled up to produce large amount of elongated particles thanks to a possible automatization [44]. As described above, the method implies basically: (i) the dispersion of preformed spherical nanoparticles (supposed to eventually contain a drug if any pharmaceutical application are foreseen) into a polymeric material film, followed by the stretching of the nanoparticles containing material at a high temperature (generally above $120^{\circ} \mathrm{C}$ ). At the end of the stretching process, the stretched particles need to be extracted from the polymeric material film $[6,7,11]$. In the present work, our aim was to set conditions for the application of the method to the elaboration of elongated nanoparticles made of biodegradable polymer. It was also aimed to apply the method at a much lower temperature $\left(50^{\circ} \mathrm{C}\right)$ as those used in previous work $\left(>120^{\circ} \mathrm{C}\right)[6,7,11]$. 
Although in previous work, the polymer films in which the nanoparticles were incorporated to be stretched were formulated in home from solution of poly(vinyl alcohol), for this work, we have selected a water soluble and stretchable film available on the market as the material to prepare the film incorporating the spherical nanoparticles to be stretched. The paper reports the different steps of the preparation of the film loaded with nanoparticles from the material of the marketed film and results on the application of the stretching method to prepare elongated nanoparticles of chitosan coated poly(isobutylcyanoacrylate) nanoparticles.

\section{Materials and methods}

\subsection{Materials}

Chitosan (CS, MW 20 kDa, deacetylation degree 92\% was purchased from Amicogen.Inc. (Jinju, South Korea), isobutylcyanoacrylate (IBCA) was gift from Henkel Biomedical (Loctite, Dublin, Ireland); a poly(vinylalcohol) (PVA) based water soluble polymer film (STBT $\left.{ }^{\circ} 30 \mu \mathrm{m}\right)\left(\mathrm{STBT}^{\circ}\right)$ was purchased from Soluble Technology Ltd. (Chechire, United Kingdom) for the elongation experiments. Milli- $Q^{\bullet}$ water was produced using the Milli- $Q^{\circ}$ direct 8 water purification system from Merck Millipore (Millipore SAS, Guyancourt, France).

\subsection{Methods}

\subsubsection{Preparation of the parent spherical nanoparticles.}

Poly(isobutylcyanoacrylate) nanoparticles coated with chitosan (CSPIBCA) were prepared by redox radical polymerization according to a previously described protocol [26,30,43]. Briefly, chitosan $(130$ $\mathrm{mg}$ ) was dissolved in $\mathrm{HNO}_{3} 0.2 \mathrm{M}(8 \mathrm{~mL})$ at $42^{\circ} \mathrm{C}$ under vigorous stirring and argon atmosphere. Once the dissolution was completed, a solution of ammonium cerium (IV) nitrate $\left(8 \times 10^{-2} \mathrm{M}\right)$ in $\mathrm{HNO}_{3} 0.2 \mathrm{M}$ $(2 \mathrm{~mL})$ and IBCA $(0.5 \mathrm{~mL})$ were added successively, the argon flux was stopped and the reaction was allowed to continue at $42^{\circ} \mathrm{C}$ for $50 \mathrm{~min}$. Then, the nanoparticles were purified by dialysis (cut-off $100,000 \mathrm{~g} / \mathrm{mol}$, Spectra/Por membranes) three times against Milli- $\mathrm{Q}^{\circ}$ water for $1 \mathrm{~h}$ and once overnight. The obtained CSPIBCA nanoparticle dispersion was stored at $4^{\circ} \mathrm{C}$ until use.

The concentration in nanoparticles of the dispersion was evaluated by gravimetry performed by measuring the mass of the dried residue obtained after lyophilisation of $0.5 \mathrm{~mL}$ of sample. The experiment was done in triplicate.

The mean hydrodynamic diameter of the nanoparticles was determined by dynamic light scattering using a Zetasizer ${ }^{\circ}$ ZS90 (Malvern Instrument, Orsay, France). Measurements were performed at $25^{\circ} \mathrm{C}$ at a scattered angle of $90^{\circ}$. Samples were diluted in Milli- $Q^{\circ}$ water to a concentration of $0.35 \mathrm{mg} / \mathrm{mL}$. 
Results were expressed as the mean hydrodynamic diameter deduced from size measurements performed on 3 different preparations of nanoparticles.

The $\zeta$ potential was deduced from the electrophoretic mobility of the particles measured by Laser Doppler Electrophoresis after dilution of CSPIBCA suspension in $\mathrm{NaCl}$ solution (1 mM, 1/100 (v/v)). Determination was achieved using a Zetasizer ${ }^{\circ}$ ZS90 (Malvern Instrument, Orsay, France).

\subsubsection{Preparation of the polymer film incorporating the nanoparticles}

At first, STBT $^{\circ}$ film (100 mg) was dissolved in $1.5 \mathrm{~mL}$ of Milli- ${ }^{\circ}$ water, under magnetic stirring for 4 hours at room temperature. The concentration of the solutions was $67 \mathrm{mg} \cdot \mathrm{mL}^{-1}$. Then, a dispersion of CSPIBCA nanoparticles at the concentration in nanoparticles of $31 \mathrm{mg} \cdot \mathrm{mL}^{-1}$ was added to this solution to a weight percentage of nanoparticles and STBT ${ }^{\circ}$ of $10 \%(323 \mu \mathrm{L}), 13 \%$ (419 $\mu \mathrm{L}$ ) and 15\% $(484 \mu \mathrm{L})$. The resulting mixture was gently stirred for $30 \mathrm{~min}$. The mixture STBT ${ }^{\circ}$-CSPIBCA was spread on a glass slide $\left(25 \times 75 \mathrm{~mm}^{2}\right)$ and dried in an incubator (Memmert, Grosseron, France) at different temperatures, 25,37 and $50{ }^{\circ} \mathrm{C}$ for $24 \mathrm{~h}$, to allow the evaporation of the water and the formation of a new film of STBT ${ }^{\circ}$ incorporating the CSPIBCA nanoparticles. For control experiments films were prepared by the same procedure but without nanoparticles. The films were used immediately after preparation

\subsubsection{Characterization of film properties}

Films containing various amounts of nanoparticles $(0,10,13,17,20 \% \mathrm{w} / \mathrm{w})$ and prepared at different temperatures $\left(25^{\circ} \mathrm{C}, 37^{\circ} \mathrm{C}, 50^{\circ} \mathrm{C}\right.$, ) were obtained according to the procedure described in 2.2.2. At first, the homogeneity of the dispersion of nanoparticles in the polymer solution was appreciated by visual inspection and under light microscopy using the contrast phase mode (Olympus BH2). Pictures were recorded using a Mightex USB2.0 color camera. After the film was formed, the homogeneity of the films was also appreciated by the same method. The purpose of these observations was to check for the occurrence of phase separation. All systems that appeared heterogenous were discarded.

Only homogenous films were selected to determine the percent elongation at break using a TA.XT2 texture analyser (Stable Micro Systems, London, United Kingdom). Films with same dimensions as the ones used to prepare elongated nanoparticles ( $25 \times 75 \mathrm{~mm}, 30 \mu \mathrm{m}$ thick) were tightly attached to grips on the TA.XT2 machine. The initial grip separation was checked prior to the start of each experiment. It corresponded to the initial length of the sample $\left(L_{0}\right)$. The cross head was moving at a rate of $0.1 \mathrm{~mm} \cdot \mathrm{s}^{-1}$ while the stretching experiment was performed at room temperature $\left(20^{\circ} \mathrm{C}\right)$ until breakage of the film. The curves gave the tensile strength (TS) as the function of the strain. The TS 
was calculated dividing the measured force, $F$ (in newton), by the section of the film, s (in $\mathrm{m}^{2}$ ) (Equation 1).

$T S=\frac{\mathrm{F}}{\mathrm{S}}$

Equation 1

The strain was deduced from the ration between the extension length, $\Delta L$, and the initial length $\left(L_{0}\right)$ (Equation 2).

Strain $=\frac{\Delta \mathrm{L}}{\mathrm{L}_{0}} \quad$ Equation 2

The elongation at breakage (EAB) was calculated following equation (3).

$E A B(\%)=\frac{\Delta L_{A B}}{L_{0}} \times 100 \quad$ Equation 3

Where $\Delta L_{A B}$ was the extension length at breakage. Measurements were performed at least 3 times on each film.

The maximal elongation $\left(E_{c_{\max }}\right)$ capacity of the film could be calculated from the equation (4).

$E_{c_{\max }}=\frac{\left(L_{0}+\Delta L_{A B}\right)}{L_{0}} \quad$ Equation 4

\subsubsection{Elongation of the film producing elongated nanoparticles}

To achieve preparation of elongated nanoparticles (E(CDPIBCA) nanoparticles), films were prepared with a concentration of $13 \%$ nanoparticles as explained in section 2.2.3. Second generation films recovered from the glass slide were placed in a home-made stretching machine as described in Figure 1. The initial grip separation length $\left(L_{0}^{\prime}\right)$ was $65 \mathrm{~mm}$. The stretching machine was placed in an incubator (Memmert, Grosseron France) at $50^{\circ} \mathrm{C}$ and let to equilibrate for $30 \mathrm{~min}$. The film was stretched at a temperature of $50^{\circ} \mathrm{C}$ and at a speed of $0.5 \mathrm{~mm} \cdot \mathrm{min}^{-1}$ to a final length, $L$, of $150 \mathrm{~mm}$ giving an elongation capacity, $E_{c}$, of $231 \%$ as calculated from equation 5 .

$E_{c}=\frac{L}{L_{0}^{\prime}} \quad$ Equation 5

Immediately after the stop of the stretching, the film was rapidly cooled down to $20^{\circ} \mathrm{C}$ using a flux of cold air (temperature $-16.5^{\circ} \mathrm{C}$ ) while it was maintained on the maximum stretched position. Then, the parts of films used to secure the film in the elongation machine were discarded (Figure 1). Effectively elongated parts were kept to recover nanoparticles embedded in the stretch film.

\subsubsection{Recovery of E(CSPIBCA) nanoparticles from the films after stretching}

The stretch part of the film was dissolved in $500 \mu \mathrm{L}$ of water or nitric acid $0.2 \mathrm{M}(\mathrm{pH}$ 1) for 30 minutes at room temperature under gentle stirring. After complete dissolution of the film, E(CSPIBA) nanoparticles were purified by Size Exclusion Chromatography (SEC) using a column of Sephacryl ${ }^{\circ}$ S1000 gel $(5 \mathrm{~cm} \times 1.4 \mathrm{~cm})$. The column was equilibrated in acetate buffer at $\mathrm{pH} 3.2$. Elution was 
performed using the same acetate buffer. The presence of nanoparticles in the eluate was detected from the turbidity and the reactivity with ninhidrin that was used to reveal the presence of amino groups of chitosan in fractions containing nanoparticles. The dispersions of purified E(CSPIBCA) nanoparticles were stored at $+4^{\circ} \mathrm{C}$.

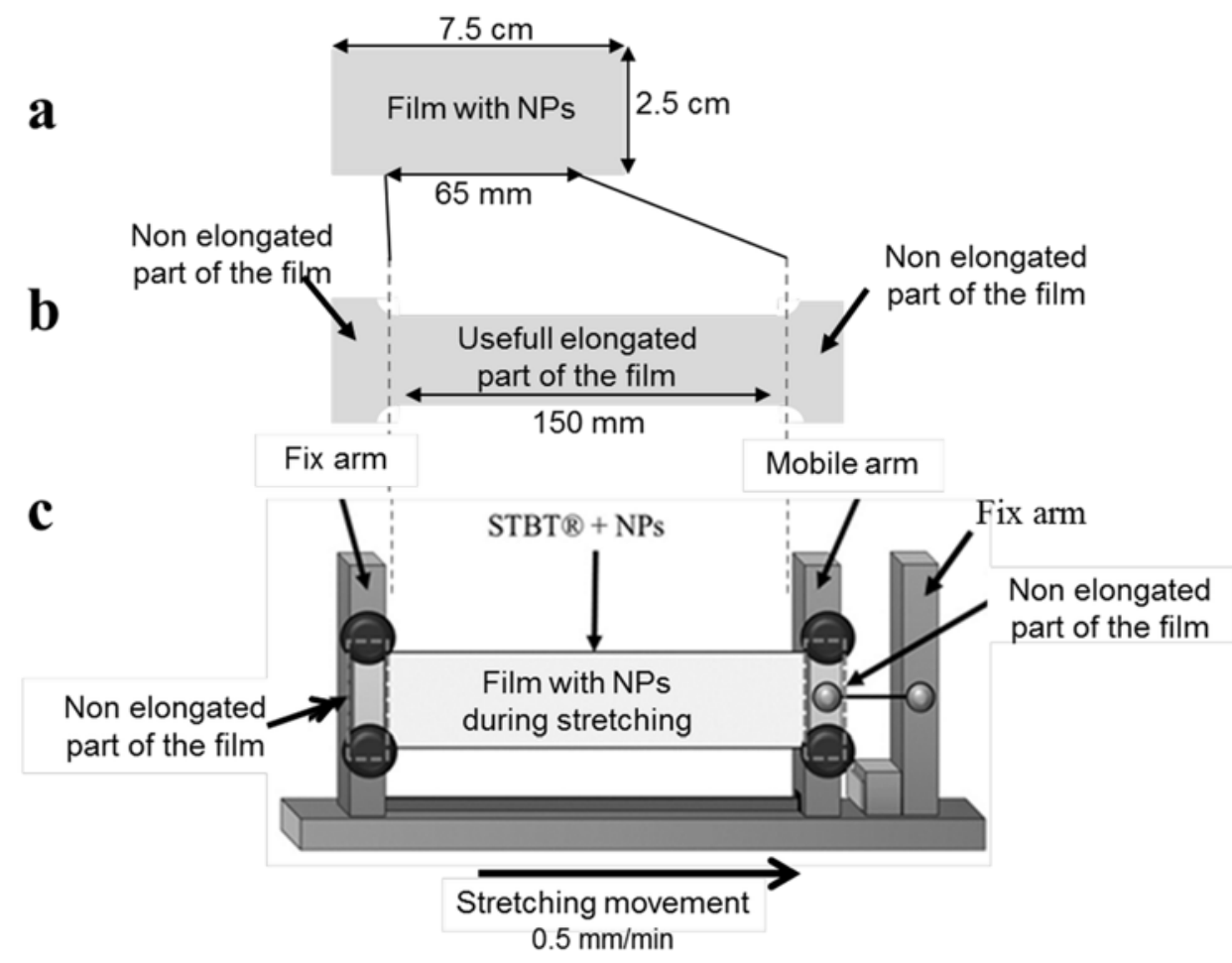

Figure 1. Scheme of the film before (a) and after (b) stretching and placed in the elongation machine (c).

\subsubsection{Characterization of the elongated nanoparticles by Transmission Electron Microscopy.}

The morphology of the nanoparticles was investigated by transmission electron microscopy (TEM). The nanoparticle dispersion $(50 \mu \mathrm{L})$ was dropped on the grid, let to dry and the nanoparticles were stained with a solution of phosphotungstic acid at $1 \%(\mathrm{pH} 7.3)$ for 5 minutes. Electron micrographs were acquired using an electron microscope JEOL 1400 MET operating at $80 \mathrm{kV}$ (Electron Microscopy Facility of I2BC, CNRS, Gif sur Yvette, France) equipped with a high-resolution digital CCD Gatan digital camera (11 megapixels). The nanoparticles were characterized by measuring their long and short axis. Measurements were performed on 300 nanoparticles to determine the shape factor (SF) defined as the ratio between the length of the long and short axis (Figure 2).

\subsubsection{Statistical analysis}

Statistical comparisons were performed utilizing analysis of variance (ANOVA) followed by the Tukey post hoc tests (GraphPad Prism v. 4 forWindows, Graph-Pad Software, San Diego, CS). Differences were considered statistically significant at $p<0.05$. 


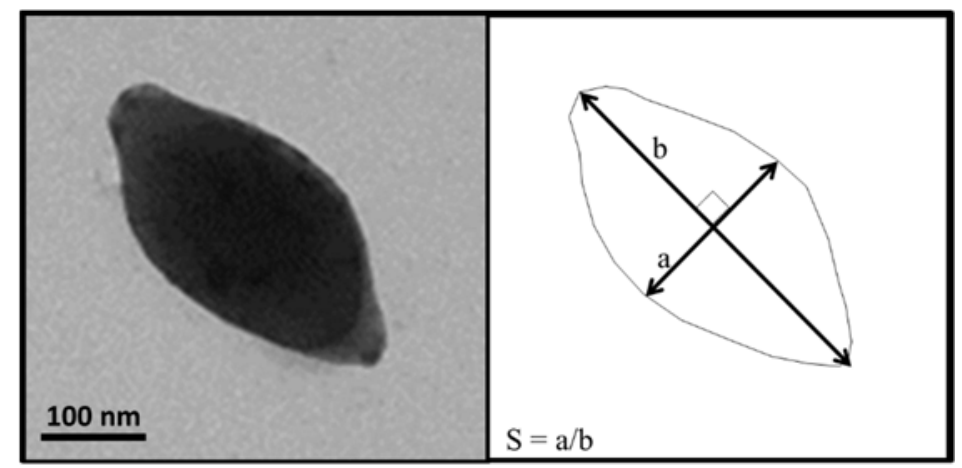

Figure 2. Calculation of the shape factor from TEM images of the E(CSPIBCA) nanoparticles.

\section{Results and discussions}

Successful production of elongated nanoparticles from parent spherical nanoparticles by the stretching method depends on the achievement of several experimental steps. At first, the parent nanoparticles need to be dispersed in a solution of polymer that will then be used to produce a stretchable film. During this step neither flocculation nor coagulation phenomena should occur. Second, colloidal stability of the nanoparticles is needed during film formation. The film should show a homogenous dispersion of the nanoparticles in the volume of the polymer film. Then, the film should be stretchable in reasonable conditions of temperature so that they will be suitable to further apply the method to particles loaded with a wide range of drugs including fragile molecules. After stretching, the stretched nanoparticles should be recovered after the dissolution of the film in water. The E(CSPIBCA) nanoparticles were prepared from parent CSPIBCA nanoparticles that occur as spherical nanoparticles and are obtained by emulsion polymerization of isobutylcyanoacrylate. Several reasons were behind the choice of the nanoparticles used in this study. At first, they are part of a large family of drug carriers that have shown interests for a broad range of therapeutic applications and considering many types of drugs [31-36]. More specifically, the CSPIBCA nanoparticles efficiently delivered anti-tumoral siRNA to tumor after intravenous administration at a distant place from the implanted tumor $[37,38]$. In this case, the siRNA was associated with the nanoparticles after preparation of the carrier. So the preparation of non-loaded nanoparticles with an elongated shape could be of interest for future studies aiming to achieve in vivo delivery of siRNA. Secondly, the parent nanoparticles have already been extensively characterized. Importantly, they are composed of a PIBCA-chitosan copolymer in which the PIBCA moiety composed the core of the nanoparticles and serve as anchor for the chitosan chains that arranged on the core surface to form a corona [30]. In that way, chitosan is covalently anchored on the surface of the nanoparticles. All soluble components that eventually remained after the synthesis of the particles were removed during the purification step. The marketed polymer film STBT ${ }^{\circ}$ was selected because it was claimed 
to dissolve rapidly in cold water and to be stretchable. Its composition based on PVA would be of good compatibility for a use in a pharmaceutical process. Water solubility and fast dissolution were mandatory characteristics. It was intended to incorporate nanoparticles within a film of polymer prepared from the material recovered from the dissolution of the marketed STBT ${ }^{\circ}$ film to which the nanoparticles were added. So, these characteristics were needed to incorporate the nanoparticles in the film and also to be able to recover the nanoparticles embedded in the film after stretching. Viscoelastic properties of the marketed film were requested to have a suitable matrix to stretch the embedded nanoparticles. According to the supplier, the STBT ${ }^{\circ}$ film has elongation capacities of $185 \%$ at room temperature and $250 \%$ at $50^{\circ} \mathrm{C}$. Besides, the marketed film showed another interesting property for the intended application. Its transparency was found useful to observe eventual occurrence of aggregation of nanoparticles introduced in the film made from the marketed film material.

The work included the following 3 steps:

- Determination of the maximal load in nanoparticles that can be incorporated in the film.

- Production of elongated nanoparticles

- Determination of the shape factor of the nanoparticles recovered from the film after elongation.

\subsection{Characteristics of the parent nanoparticles}

The parent CSPIBCA nanoparticles used in the present study consisted of core-corona type nanoparticles. The core was composed of PIBCA that was coated by a layer of chitosan covalently anchored to the nanoparticle core by PIBCA chains $[30,38]$. Characteristics of the nanoparticles synthesized for this work were consistent with those of our previous works $[\mathbf{2 8 , 3 0 , 3 8}]$ with a mean hydrodynamic dynamic diameter of $394 \pm 1 \mathrm{~nm}$ and a zeta potential of $+22.7 \pm 0.6 \mathrm{mV}$ evaluated at neutral $\mathrm{pH}$. The concentration of the parent dispersion was $31 \mathrm{mg} \cdot \mathrm{mL}^{-1}$. The number of nanoparticles, $N_{p}$, found in $1 \mathrm{mg}$ can be calculated from the hydrodynamic diameter of the nanoparticles converted in $\mathrm{cm}, \mathrm{d}_{\mathrm{H}}$, and the volumetric mass, $\rho$ that is $1140 \mathrm{mg} \cdot \mathrm{mL}^{-1}$ for poly(isobutylcyanoacrylate) nanoparticles [45] (Equation 6).

$N_{p}=\frac{6}{\rho \pi d_{H}^{3}} \quad$ Equation 6

By applying equation 1, it could be determined that one $\mathrm{mg}$ of nanoparticles contained $27.410^{9}$ nanoparticles. 


\subsection{Determination of the maximal concentration in nanoparticles that can be incorporated in the film.}

It is known that colloids can flocculate or coagulate in the presence of polymers [46]. Thus, the first step of the work was dedicated to the preparation of films incorporating nanoparticles in which the nanoparticles would be homogenously dispersed while the new film would show interesting stretching properties for the intended application. As indicated by the supplier, the STBT ${ }^{\circ}$ film dissolved easily in water. A solution of the polymer obtained from the dissolution of the film could be prepared at a concentration of $67 \mathrm{mg} \cdot \mathrm{mL}^{-1}$. Addition of nanoparticles to the polymer solution led to homogenous dispersions from both visual and microscopic observations up to a load in nanoparticles of $15 \%$ expressed as the percentage of nanoparticles in weight added from the weight of polymer dissolved. Above this load, a phase separation was observed. Films were prepared only with nanoparticle containing dispersions that appeared homogenous. Films prepared at room temperature and at $50^{\circ} \mathrm{C}$ showed the occurrence of a phase separation (Figure 3). Conversely, films obtained from a working procedure carried out at $37^{\circ} \mathrm{C}$ were homogenous at both macroscopic and microscopic scales up to a load in nanoparticles of $13 \%$ (Figure 3). For a film obtained from a solution composed of $\mathrm{STBT}^{\circ}$ at a concentration of $67 \mathrm{mg} \cdot \mathrm{mL}^{-1}$ and nanoparticles at a concentration of 6.8 $m g \cdot \mathrm{mL}^{-1}$ (10\% load), the calculated number of nanoparticles per unit surface area dispersed in the film, $\mathrm{n}$, was $14.5 \times 10^{9}$ nanoparticles. $\mathrm{cm}^{-2}$. This number could be calculated from equation 7 with $\mathrm{N}_{\mathrm{p}}$, the number of nanoparticles in $1 \mathrm{mg}$ deduced from equation $6, \mathrm{v}$, the volume of dispersion of nanoparticles added during the preparation of the film $(0.323 \mathrm{~mL}), \mathrm{C}$ the concentration in nanoparticle of the dispersion (31 mg. $\left.\mathrm{mL}^{-1}\right)$ and the area of the film, $\mathrm{A}\left(2.5 \times 7.5 \mathrm{~cm}^{2}\right)$.

$n=\frac{\mathrm{Cv} N_{p}}{\mathrm{~A}} \quad$ Equation 7

Only films that appeared homogeneous under the microscope were further used. Obviously, embedding nanoparticles into the films could considerably influence their mechanical and viscoelastic characteristics, compared to a nanoparticle-empty STBT ${ }^{\circ}$ films. The stretching characteristics of the films, both unloaded and embedding nanoparticles, could be a critical determinant of the elongation efficacy. Therefore, the maximal elongation at breakage, $E A B$, of the films prepared with various loads of nanoparticles was determined using a universal mechanical testing machine (Texture analyser). The figure 4a presents tensile stress of the films monitored during the elongation process. Consistently to what was expected, the tensile stress increased up to a maximum where the curves showed a sudden break that corresponded to the breakage of the film. The general shape of the curves suggested that the films behaved like a ductile material. The breakage occurred in the plastic domain of the film. 


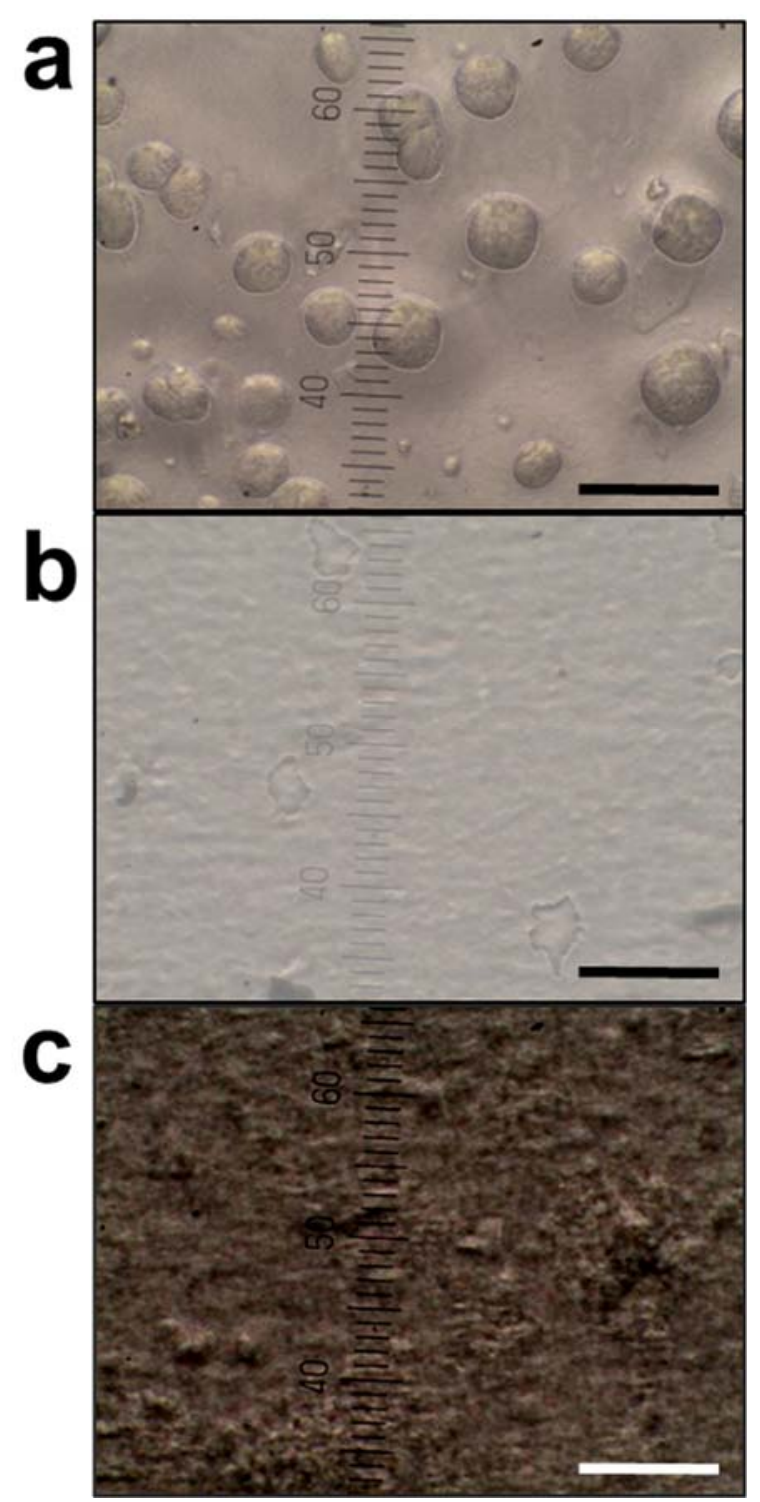

Figure 3. Optic micrographs of the films obtained with $13 \%$ nanoparticles after drying at room temperature (a), $37^{\circ} \mathrm{C}(\mathrm{b})$ and $50^{\circ} \mathrm{C}(\mathrm{c})$. The films dried at room temperature and $50^{\circ} \mathrm{C}$ showed clear phase separation while the film dried at $37^{\circ} \mathrm{C}$ appeared homogenous. Scale bar: $100 \mu \mathrm{m}$.

The maximum elongation determined at breakage, $\Delta L_{A B}$ was used to calculate the $E A B$ assessing the stretchable characteristics of the films loaded with different percentage of nanoparticles. Incorporation of nanoparticles in the films reduced the EAB in comparison with measurements performed on a film prepared without nanoparticles. The decrease was moderate with films loaded with 10 and $13 \%$ of nanoparticles and was not significantly different between the two-film load. The decrease was more important for the film loaded with $15 \%$ of nanoparticles and significantly different from the films loaded with 10 and 13\% nanoparticles (figure $4 \mathrm{~b}$ ). This result was not so surprising as the addition of particles in films of polymer can create defaults that may induce the breakage at lower extension length compared with a film produced in the same conditions without a load of particles. 

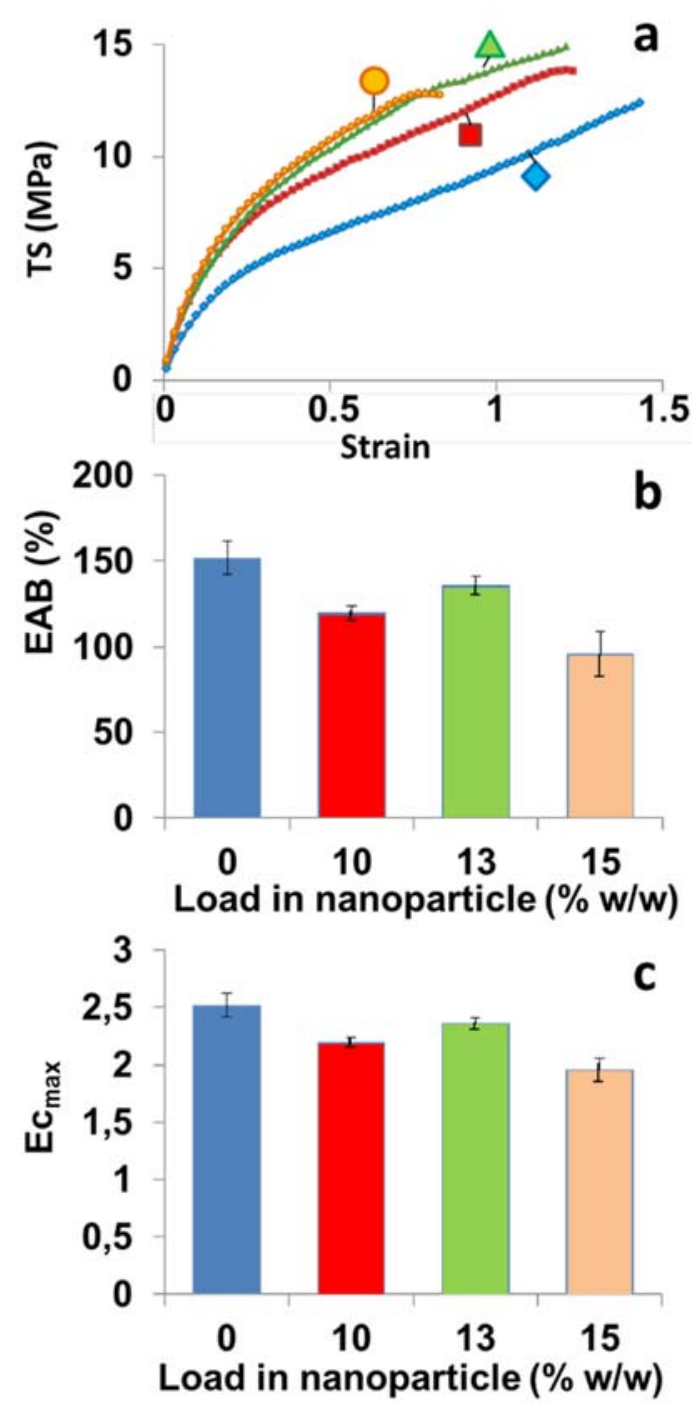

Figure 4. Elongating properties of the STBT film loaded with different weight percentages of CSPIBCA nanoparticles. (a) Exemple of tensile strength (TS) of films monitored during stretching and recorded with a texture analyser (load in nanoparticles expressed in weight percentage of STBT ${ }^{\circ}: 0 \%$ (bleu diamonds), 10\% (red squares), 13\% (green triangles), 15\% (orange circles), (b) Maximal elongation at breakage (EAB) expressed as a percentage from the initial length ( $\left.L_{0}\right)$ of the film, (c) Maximal elongation capacity $\left(E c_{\max }\right)$ of the film as determined from the total length of the film at breakage divided by the initial length (Lo). Statistics: No significant difference was found comparing STBT ${ }^{\circ}$ films loaded with 10 and $13 \%$ nanoparticles.

A compromise between the maximal concentration in nanoparticles in the film and the elongation property was adopted to produce elongated nanoparticles. The choice was guided giving the priority to keep at a maximum the elongation property of the film. Thus, the film loaded with $13 \%(\mathrm{w} / \mathrm{w})$ of nanoparticles was chosen as its $E A B$ was evaluated at $136 \pm 5 \%(n=3)$. It can be calculated from equation 4 that the theoretical maximal elongation capacity of this film was $2.36 \pm 0.05(n=3)$. Thus, it could be assumed that nanoparticles embedded and stretched in this film at its maximal elongation capacity could reach a maximal theoretical value of their shape factor of 2.36 . 


\subsection{2. Production of elongated nanoparticles.}

The selected film loaded with $13 \%$ nanoparticles was placed in the stretching machine to be stretched at $50^{\circ} \mathrm{C}$. The machine was set to perform an elongation of the film giving more than twice its original length (230\%) but remaining slighly below the $E A B$ predetermined for this film to avoid film breakage. At the end of the experiment, it was assumed that a tempering of the film would be necessary to preserve the new shape acquired by the nanoparticles that were submitted to the elongation stress. Thus, films were cooled down immediately after the elongation was stopped by blowing cold air $\left(-16^{\circ} \mathrm{C}\right)$ and allowing the temperature of the film to drop down to the ambient temperature in less than $30 \mathrm{sec}$. The effective experimental elongation that was calculated from the measurement of the length of the film before and after recovery from the elongating machine was 2.3 consistently with the applied operating conditions.

Nanoparticles embedded in the film were then recovered by dissolution of the film in water and observed by TEM to evaluate the efficacy of the stretching procedure (Figure 5). Nanoparticles recovered from stretched films after dissolution in pure water showed an elongated shape (Figure 5b). Parent nanoparticles recovered from non-stretched films appeared spherical (Figure 5a). In both cases, particles recovered from the dissolution of films were embedded in a foreign material and the background of the preparation observed in microscopy appeared very dusty that was unusual for these nanoparticles. Observations of a solution obtained from the dissolution of a piece of film that was prepared without nanoparticles revealed the presence of insoluble material occurring as more or less aggregated particles suggesting that the film did not dissolve totally in the experimental condition used for this study (Figure 6). The material of the dusty background that remained on the nanoparticle surface after extraction from the film was attributed to this insoluble material. A purification step was needed to clean nanoparticles from contaminant material left by incomplete dissolution of the film. On the TEM image, the size of the insoluble material appeared much smaller than that of the parent CSPIBCA nanoparticles $\left(D_{H}=394 \pm 1 \mathrm{~nm}\right.$ as evaluated by dynamic light scattering) suggesting that it could be separated by size exclusion chromatography provided that it was removed from the nanoparticle surface. Evaluation of the zeta potential of CSPIBCA nanoparticles as obtained after synthesis and of the particles formed of the insoluble material obtained after dissolution of the film revealed that the two types of particles had an opposite value of zeta potential at neutral $\mathrm{pH}$. While the CSPIBCA nanoparticles showed a positive zeta potential of $+22.7 \pm 0.6 \mathrm{mV}$, that of the insoluble particles of the STBT ${ }^{\circ}$ film was negative at $-1.0 \pm 0.1 \mathrm{mV}$ (Figure 7a). 

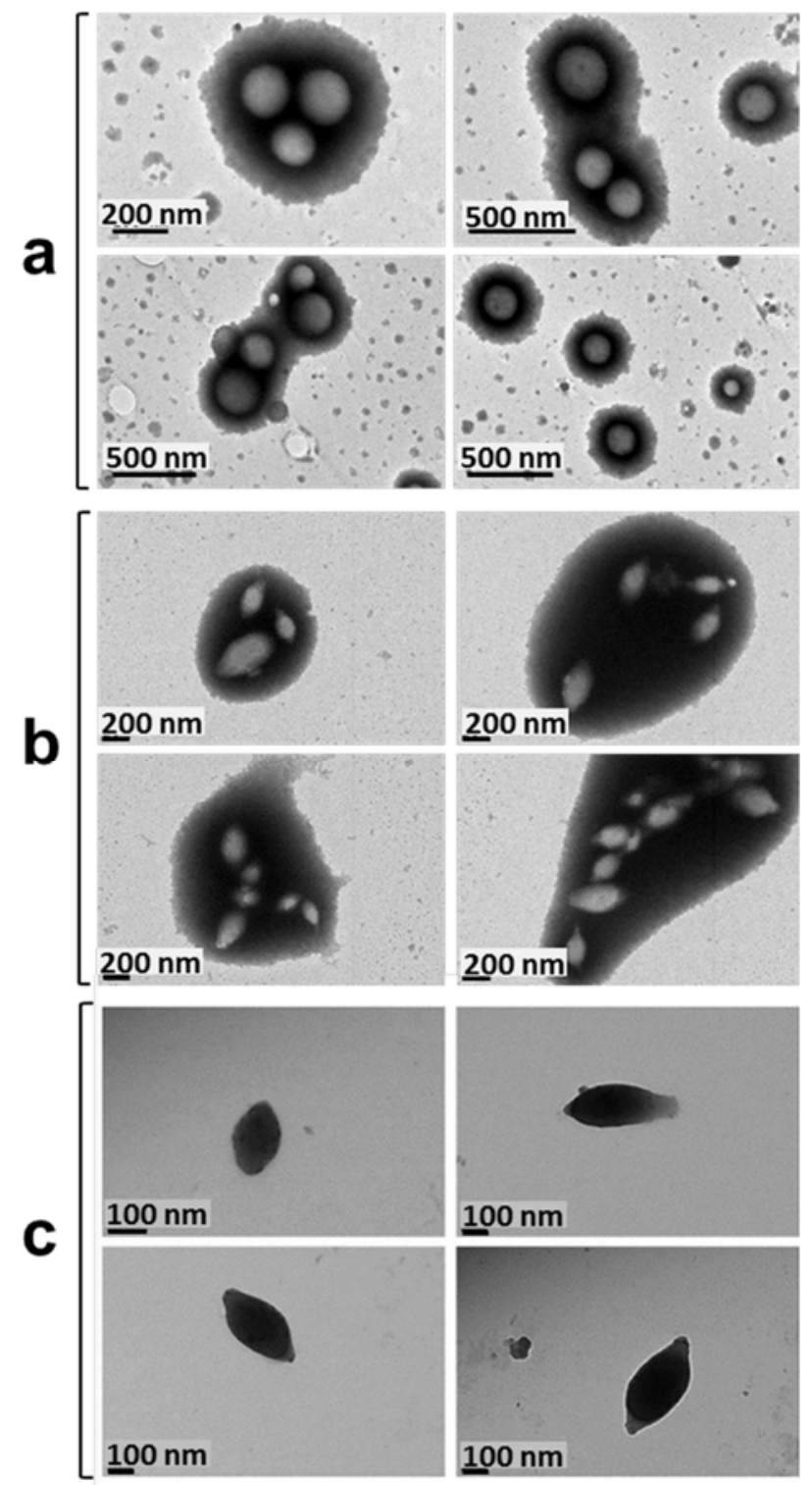

Figure 5. Transmission Electron Microscographs (TEM) of the parent CSPIBCA nanoparticles (a) and of the E(CSPIBCA) nanoparticles recovered after dissolution of the film in pure water (b). E(CSPIBCA) nanoparticles recovered from the film after dissolution at $\mathrm{pH} 1$ and purification by SEC performed at $\mathrm{pH} 3.5$ (c).

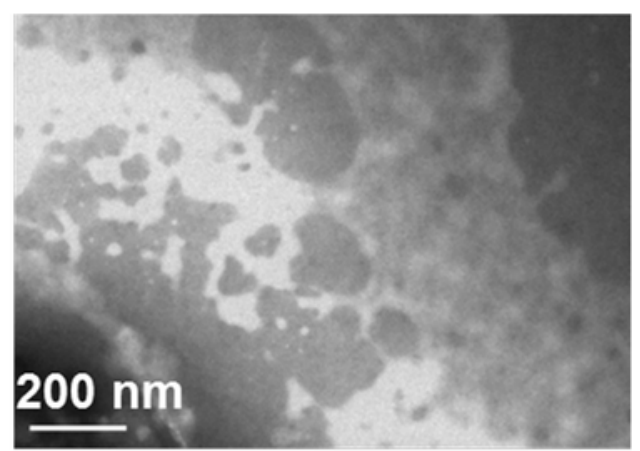

Figure 6. Transmission electron micrographs of $S T B T^{\circ}$ film after dissolution in water. 


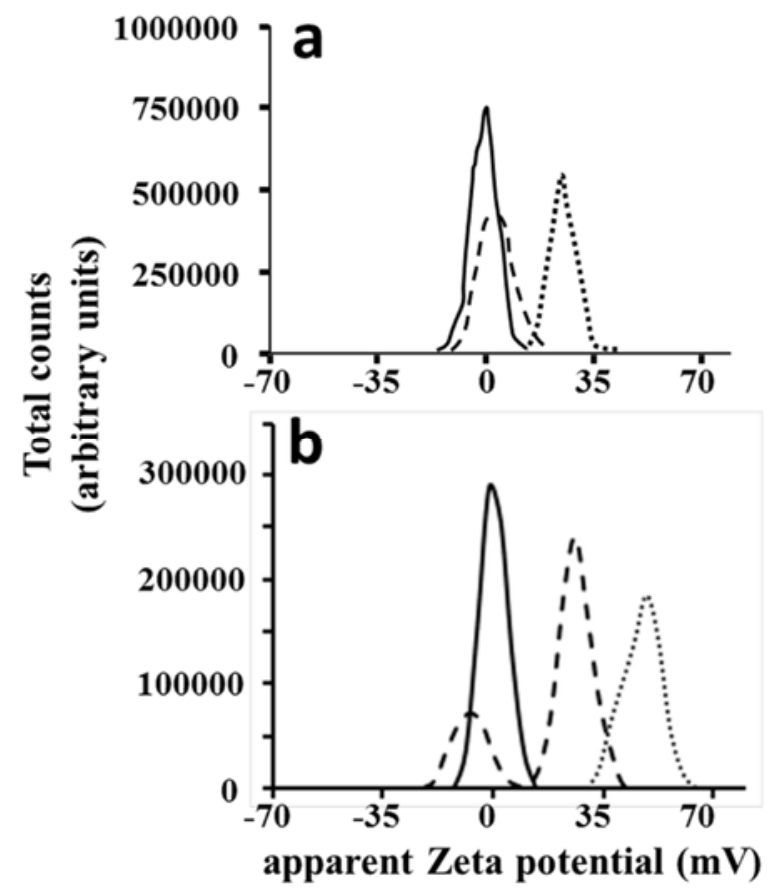

Figure 7. Zeta potential of the insoluble material remaining after dissolution of the STBT ${ }^{\circ}$ film (solid line), of the CSPIBCA parent nanoparticles (dotted line) and the dispersion recovered from the dissolution of a STBT ${ }^{\circ}$ film loaded with CSPIBCA nanoparticles (semi solid line). (a) Dispersions were prepared at $\mathrm{pH}$ 7, (b) dispersions were prepared at $\mathrm{pH} 1$.

Opposite values of zeta potential could explained the observed accumulation of contaminant material on the surface of CSPIBCA nanoparticles recovered from the dissolution of the film on the TEM images (Figure 5a,b). Consistently, zeta potential evaluated on particles recovered after dissolution of the film showed a single peak with an almost neutral value of zeta potential (Zeta potential $=+2.9 \pm 0.2 \mathrm{mV}$ ) that was close to the one of the insoluble particles of the STBT ${ }^{\circ}$ film. By decreasing the $\mathrm{pH}$ down to a value of 3.2 , particles from the insoluble part of the film became neutral (Zeta potential $=+0.3 \pm 0.4 \mathrm{mV}$ ). At this $\mathrm{pH}$, the zeta potential of the CSPIBCA based nanoparticles was still frankly positive (Zeta potential $=+50.5 \pm 2.9 \mathrm{mV}$ ). Evaluation of the zeta potential of a dispersion obtained from the dissolution of a film loaded with CSPIBCA nanoparticles at this $\mathrm{pH}$ revealed two peaks which values corresponded to the insoluble particles of the STBT film (Zeta potential $=+7.1 \pm 5.6 \mathrm{mV}$ ) and to the CSPIBCA nanoparticles (Zeta potential $=+26.4 \pm 5.3 \mathrm{mV}$ ) respectively (Figure $7 \mathrm{~b}$ ). It is noteworthy that the zeta potential of the CSPIBCA nanoparticles recovered after dissolution of the film (Zeta potential $=+26.4 \pm 5.3 \mathrm{mV}$ ) was very closed to that of the CSPIBCA nanoparticles before their incorporation into the film $(+22.7 \pm 0.6 \mathrm{mV})$. This indicated that the surface of the nanoparticles recovered from the film was not chemically modified by the incorporation in the film and the recovery method. This was consistent with the fact that chitosan 
was stably bound on the nanoparticle surface thanks to a covalent anchor of PIBCA that composed the core of the nanoparticles $[\mathbf{3 0 , 3 8}]$. The nanoparticles could be purified by SEC after dissolution at acidic $\mathrm{pH}$ and performing the separation at $\mathrm{pH}$ 3. The nanoparticles were eluted at first as revealed by the purple colour developed in the presence of ninhidrine acknowledging the presence of the CSPIBCA nanoparticles. The contaminant particles produced during the process of dissolution of the film were eluted later (Figure 8). The purification was efficient to purify the nanoparticles as suggested by the TEM images that showed perfectly isolated and clean nanoparticles (Figure $5 c$ ). TEM images also provided evidence of the presence of chitosan on the surface of the E(CSPIBCA) nanoparticles. It is shown as a less dense to electrons layer on the surface of the core of the nanoparticles that appears denser to electrons on the TEM images (Figure 2, Figure 5c).

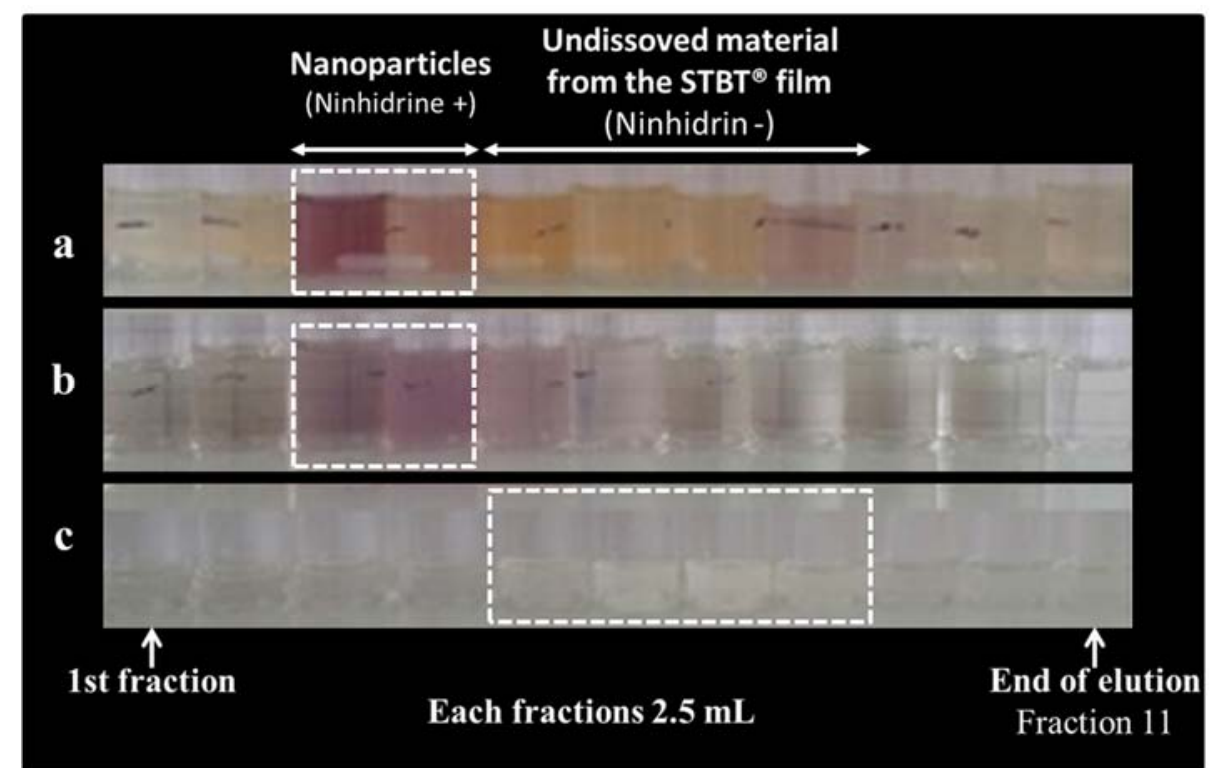

Figure 8. Purification of the E(CSPIBCA) nanoparticles by size exclusion chromatography on Sephacryl $S-1000$ gel. Elution of the material contained in the dispersion obtained after dissolution of an elongated film loaded with nanoparticles (a), Elution of purified E(CSPIBCA) nanoparticles (b) and of the insoluble material recovered after dissolution of the STBT ${ }^{\circ}$ film (c).

\subsection{Determination of the shape factor of the nanoparticles recovered from the film after elongation.}

The elongated nanoparticles exhibited an oblate shape, some having a "lemon" like figure. The determination of the shape factor of the stretched nanoparticles was achieved on the samples using the maximum elongation allowed for the film before breakage. It was assumed that these conditions would provide the maximum elongation we could expect for the CSPIBCA nanoparticles in the experimental conditions used based on the nature of the film and the operating temperature. Characterizations were performed within a period of 2 weeks from the production of the elongated nanoparticles. 
The figure 9 shows the distribution of the shape factor calculated from measurements performed on TEM pictures obtained from 300 individual nanoparticles. It is noteworthy that only very few methods are suitable to evaluate morphological characteristics of elongated nanoparticles [47]. Here TEM was selected as it is one of the microscopic techniques that give direct evidence of the morphology of particles at this scale of size.

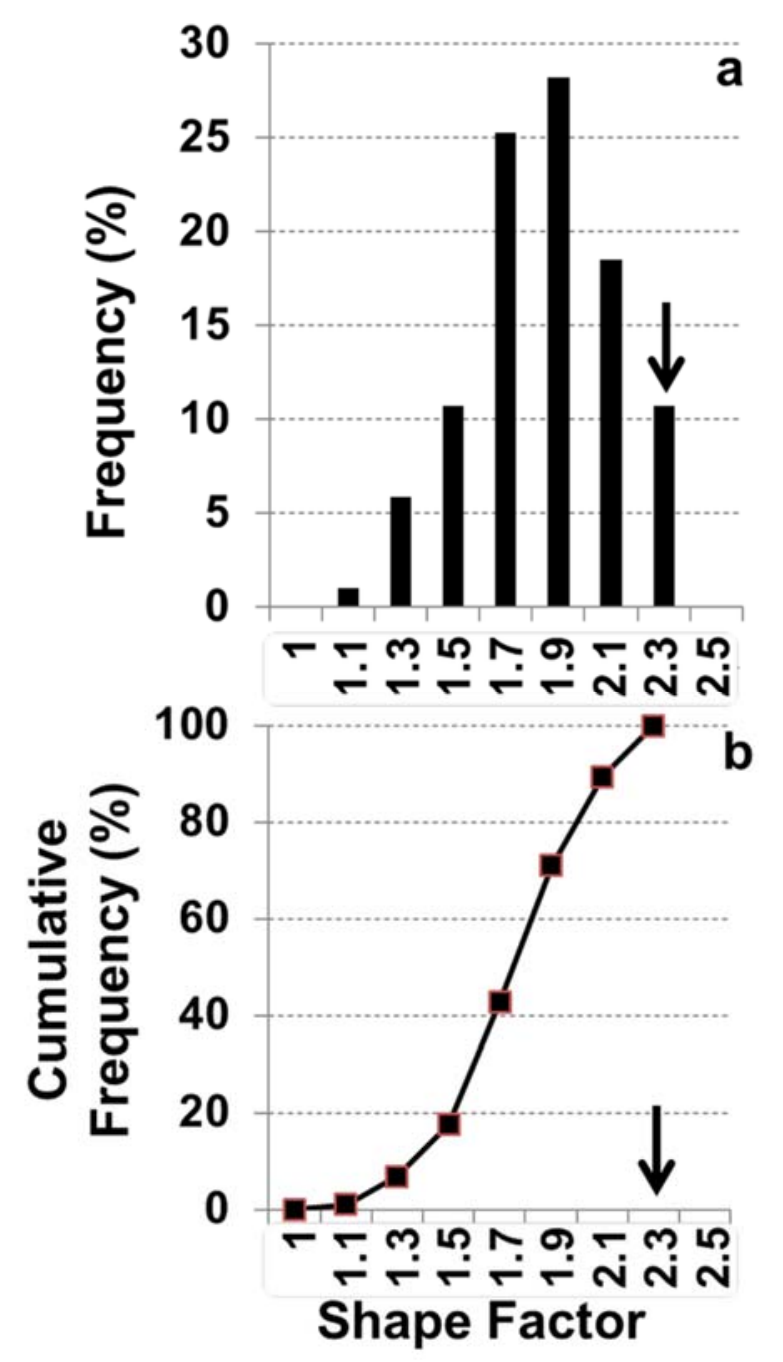

Figure 9. Distribution of the shape factor of the E(CSPIBCA) nanoparticles recovered from a films of $S T B T^{\circ}$ of $2^{\text {nd }}$ generation loaded with $13 \%$ nanoparticles (wt/wt) and stretch to an elongation of $230 \%$ at $50^{\circ} \mathrm{C}$ with the stretching machine. The nanoparticles were recovered after dissolution of the film at $\mathrm{pH} 1$ and purification by size exclusion chromatography. Measures were performed from TEM images as illustrated in figure 2.

The mean shape factor of the recovered elongated nanoparticles was of $1.76 \pm 0.29$. All nanoparticles recovered from a stretched film were elongated since none of them showed an elongation factor of 1 corresponded to spherical particles. Up to $80 \%$ of the E(CSPIBCA) nanoparticles displayed a shape factor above 1.5 and $10 \%$ of the particles were having a shape factor that corresponded to the maximal value expected (i.e., 2.3) that could be calculated from the 
elongation capacity of the film. The nanoparticles embedded in the film were significantly distorted during the stretching procedure. However, the discrepancy between the film elongation and the one of the particles suggest that micro-rheological behaviour of the CSPIBCA nanoparticles dispersed in the PVA matrix of the film, as well as the temperature and the imposed macroscopic rate of deformation could be important determinants of the attainable deformation, which would deserve further investigations.

\section{Conclusion}

The procedure proposed in the present work was suitable to prepare E(CSPIBCA) nanoparticles with a shape factor up to 2.3 working at a reasonable temperature at $50^{\circ} \mathrm{C}$ with marketed water-soluble film forming materials. Many drugs are stable at this temperature that makes this procedure suitable to be applied to produce elongated nanoparticles being loaded with a wide range of drugs. Further, film loads in nanoparticles was high (up to $13 \%$ expressed in dry weight) and the method allows the recovery of an aqueous suspension of elongated nanoparticles simply by dissolution of the watersoluble PVA film. Besides, the method was applied to prepare elongated species of poly(alkylcyanoacrylate) nanoparticles from their spherical counterparts. The elongated species will constitute a new model of nanoparticles complemental to the already available series of spherical species. They will be used in studies considering the influence of the shape of nanoparticles on their in vivo fate under various situations for their application as drug delivery systems. They will also be used to investigate the influence of the nanoparticle shape with biological systems by in vitro methods. For instance, they are of interest to investigate the influence of the nanoparticle shape on (i) nanoparticle interactions with biomacromolecules, particularly with proteins present in biological fluids, (ii) the capacity of the nanoparticles to go through biological barriers, including epithelia and endothelia as well as (iii) their propensity to be endocytosed by cells. This new model of nanoparticles will also be interested to investigate the influence of the shape of the nanoparticles in drug delivery methods that consider a mucosal route of administration.

\section{Acknowledgments}

C. Palazzo received a fellowship from "Ministero dell'Istruzione dell'Università e della Ricerca", MIUR, Italy. The present work has benefited from the facilities and expertise of the Electron Microscopy facilities of 12BC, CNRS, Gif sur Yvette, France (http://www.cgm.cnrsgif.fr/spip.php?article282\&lang=fr). 


\section{References}

1 Brown, P.D. and Patel, P.R., WIREs Nanomed. Nanobiotechnol., 7, 125-130(2015).

2 Alexis, F., Pridgen, E., Molnar, L.K. and Farokhzad, O.C., Mol. Pharm., 5, 505-15 (2008).

3 Clogston, J.D. and Patri, A.K. In: Dobrovolskaia, M., McNeil S.E. (2013). Frontiers in nanobiomaterial research $\mathrm{Vol}$ 1: Handbook of immunological properties of engineered nanomaterials, (World Scientific Publishing Co. Pte. Ltd.), pp. 25-52.

4 Wang, J., Byrne, J.D., Napier, M.E. and DeSimone, J.M., Small, 7 (2011) 1919-1931.

5 Moghimi, S.M., Hunter, A.C. and Andresen, T.L., Ann. Rev. Pharmacol. Tox., 52 (2012) 481-503.

6 Cauchois, O., Conception, Preparation \& Characterization of nanoparticles of complex shapes: Study of their in vivo fate, PhD Thesis of the Université Paris Sud - Paris XI, 2011, HAL Id: tel00659379. https://tel.archives-ouvertes.fr/tel-00659379 .

7 Champion, J.A. and Mitragotri, S., Proc. Natl. Acad. Sci. USA, 103 (2006) 4930-4934.

8 Champion, J.A., Katare, Y.K. and Mitragotri, S., Proc. Natl. Acad. Sci. USA, 104 (2007) 1190111904.

9 Barua, S., Yoo, J.W., Kolhar, P., Wakankar, A., Gokarn, Y.R. and Mitragotri, S., Proc. Natl. Acad. Sci. USA, 110 (2013) 3270-3275.

10 Mathaes, R., Winter, G., Besheer, A. and Engert, J., Int. J. Pharm., 465 (2014) 159-164.

11 Kumar, S., Anselmo, A.C., Banerjee, A., Zakrewsky, M., Mitragotri, S., J. Control. Release, 28 (2015) 141-148.

12 Vauthier, C. and Bouchemal, K., Pharm. Res., 26 (2009) 1025-1058.

13 Prasad Rao, J. and Geckeler, K.E., Prog. Polym. Sci., 36 (2011) 887-913.

14 Meyer, R.A. and Green, J.J., WIREs Nanomed. Nanobiotechnol., 8 (2016) 191-207.

15 Cauchois, O., Segura-Sanchez, F. and Ponchel, G., Int. J. Pharm., 452 (2013) 292-299.

16 Landwehr, J., Fader, R., Rumler, M., Rommel, M., Bauer, A.J., Frey, L., Simon, B., Fodor, B., Petrik, P., Schiener, A., Winter, B. and Spiecker, E., Nanotechnology, 25 (2014) 505301.

17 Beck-Broichsitter, M., Nicolas, J. and Couvreur, P., Eur. J. Pharm. Biopharm., 97 (2015) 304-17.

18 Mitragotri, S., Pharm. Res., 26 (2009) 232-234.

19 Liu, Y., Tan, J., Thomas, A., Ou-Yang, D and, Muzykantov, V.R., Ther. Deliv., 3 (2012) 181-194.

20 Chu, K.S., Hasan, W., Rawal, S., Walsh, M.D., Enlow, E.M., Luft, J.C., Bridges, A.S., Kuijer, J.L., Napier, M.E., Zamboni, W.C. and DeSimone, J.M., Nanomedicine, 9 (2013) 686-693.

21 Kolhar, P., Anselmo, A.C., Gupta, V., Pant, K., Prabhakarpandian, B., Ruoslahti, E. and Mitragotri, S., Proc. Natl. Acad. Sci. U S A, 110 (2013) 10753-10758.

22 Setyawati, M.L., Tay, C.Y., Docter, D., Stauberb, R.H. and Leong, D.T., Chem. Soc. Rev., 44 (2015), 8174-8199.

23 Truong, N.P., Whittaker, M.R., Mak, C.W. and Davis, T.P., The importance of nanoparticle shape in cancer drug delivery. Expert. Opin. Drug. Deliv. 12 (2015) 129-142.

24 Barbosa, M.E., Bouteiller, L., Cammas-Marion, S., Montembault, V., Fontaine, L. and Ponchel, G., J. Mol. Recognit., 21 (2008) 169-178.

25 Chauvierre, C., Labarre, D., Couvreur, P. and Vauthier, C., Pharm. Res., 20 (2003) 1786-1793.

26 Bertholon I., Vauthier, C., Labarre D. Pharm. Res., Pharm. Res., 23, (2006) 1313-1323.

27 Lira, M.C., Santos-Magalhães, N.S., Nicolas, V., Marsaud, V., Silva, M.P., Ponchel, G. and Vauthier, C., Eur. J. Pharm. Biopharm., 79 (2011) 162-170.

28 Bravo-Osuna I., Ponchel G. and Vauthier C., Eur. J. Pharm. Sci., 30 (2007) 143-154.

29 Nicolas, J. and Couvreur, P., Wiley Interdiscip. Rev. Nanomed. Nanobiotechnol., 1 (2009) 111-127. 
30 Zandanel, C. and Vauthier, C., J. Coll. Sci. Biotechnol., 1 (2012) 68-81.

31 Barraud, L., Merle, P., Soma, E., Lefrancois, L., Guerret, S., Chevallier, M., Dubernet, C., Couvreur, P., Trepo, C. and Vitvitski L., J. Hepatol., 42 (2005) 736-743.

32 Couvreur, P. and Vauthier, C., Pharm. Res., 23 (2006) 1417-1450.

33 Vauthier, C., Labarre, D. and Ponchel G., J. Drug Target., 15 (2007) 641-663.

34 Soma, E., Attali, P. and Merle, P., In: Alonso, M.J. and Csaba, N.S. (2012). RSC Drug Discovery Series No. 22: Nanostructured Biomaterials for Overcoming Biological Barriers, (The Royal Society of Chemistry, Cambridge, UK), pp. 591-600.

$35 \underline{\mathrm{http} / / \text { www.onxeo.com/fr/nos-produits/portefeuilles-produits/orphelins-oncologie/ }}$ Accessed 12 May 2016.

36 Zhou, Q., Sun, X., Zeng, L., Liu, J. and Zhang, Z., Nanomedicine. 5 (2009) 419-423.

37 Ramon, A.L., Bertrand, J.R., de Martimprey, H., Bernard, G., Ponchel, G., Malvy, C. and Vauthier, C., J. Mol. Recognit., 26 (2013) 318-329.

38 de Martimprey, H., Bertrand, J.R., Malvy, C., Couvreur, P., Vauthier, C., Pharm. Res., 27 (2010) 498-509.

39 Labarre, D., Vauthier, C., Chauvierre, C., Petri, B., Muller, R. and Chehimi, M.M., Biomaterials, 26 (2005) 5075-5084.

40 Vauthier, C., Lindner, P. and Cabane, B., Colloids Surf. B Biointerfaces, 69 (2009) 207-215.

41 Vauthier, C., Persson, B., Lindner, P., Cabane, B., Biomaterials, 32 (2011) 1646-1656.

42 Alhareth, K., Vauthier, C., Bourasset, F., Gueutin, C., Ponchel, G. and Moussa, F., Eur. J. Pharm. Biopharm., 81 (2012) 453-457.

43 de Martimprey, H., Vauthier, C., Malvy, C. and Couvreur, P., Eur. J. Pharm. Biopharm., 71 (2009) 490-504.

44 Meyer, R.A., Meyer, R.S., Green, J.J., J. Biomed. Mater. Res. A., 103 (2015) 2747-2757.

45 Vauthier, C., Schmidt, C., Couvreur P., J. Nanoparticle Res., 1 (1999) 411-418.

46 Kamibayashi, M., Ogura, H. and Otsubo, Y., J. Colloid Interface Sci., 321 (2008) 294-301.

47 Mathaes, R., Winter, G., Engert, J., Besheer, A., Int. J. Pharm., 10 (2013) 620-629. 